\title{
Tape measure to aid prescription in paediatric resuscitation
}

\author{
G. HUGHES, H. SPOUDEAS, I. Z. KOVAR \& \\ H. T. MILLINGTON \\ Departments of Accident and Emergency and Paediatrics, Charing Cross Hospital, \\ London, England
}

\section{SUMMARY}

A tape measure, based on 50th centile weight for height and designed to permit easy drug dosage calculation, endotracheal tube size and DC cardioversion current dosages in childrens' emergencies, was tested for reliability by medical and nursing staff with varying paediatric experience. We found that the tape measure gave a reproducible estimate of weight and suggest that its use would facilitate decision making by inexperienced medical and nursing staff in paediatric resuscitation when there is no time to weigh the child.

\section{INTRODUCTION}

The management of paediatric resuscitation should not in theory be a problem for the trained paediatrician. In the United Kingdom, however, there are few dedicated childrens' hospitals and even fewer paediatric casualty departments. Emergencies in children are largely dealt with in an adult accident and emergency environment by junior medical and nursing staff who are often inexperienced in the care of children.

Prescription of drugs in children is largely based on body weight and surface area parameters. In emergencies true weights are unobtainable and hence only estimates can be used to calculate drug and fluid therapy. These estimates can only be best guesses and are based largely on the experience of the medical and nursing staff, or derived from formulae based on age/height/body habitus (Weech, 1956; Catzel \& Oliver, 1981; Garland, 1986). There is always the potential for the occasional major error particularly in the stressful emergency situation.

Correspondence: Dr I. Z. Kovar, Department of Paediatrics, Charing Cross Hospital, Fulham Palace Road, London W6 8RF, England. 
We describe a simple and rapid tape measure method which we believe permits an accurate estimation of weight in children. The study aim was to confirm the accuracy of the tape in practice when used by different grades of medical and nursing personnelz with varied paediatric experience.

\section{MATERIALS AND METHODS}

A tape measure, graduated in kilograms body weight corresponding to the 50th percentile weight for a given height, but age independent, was used (Figure 1). The tape $\vec{\circ}$ was initially devised by Dr J. Broselow using data from 29,000 North American children (Hamil et al., 1979). Calculated drug dosages (JAMA, 1986) and recommended $\omega$ number of joules for DC countershock for each weight increment are displayed on one 3 side of the tape; recommended equipment sizes (e.g. endotrachael tube sizes) on the other.

The study was limited to a 6-week period. Patients were considered in three groups and the procedure and sequence followed in all three groups was similar. The child's weight was first estimated (by either a nurse or doctor) to the nearest $0.5 \mathrm{~kg}$ and recorded. The tape predicted weight using the tape measure stretched crown to heel for each child was then similarly recorded to the nearest kilogram. Following this the childP was weighed and measured using standard calibrated clinical scales and a stadiometer oxto measuring board, to the nearest $0.1 \mathrm{~kg}$ and 0.1 metre respectively. Tape and obserser estimated weights were plotted against true weights and surface areas calculated usingo published nomograms (Catzel \& Oliver, 1981; Shirkey, 1975).

Group 1: 61 children (aged 6 weeks to 10 years) who were seen consecutively in åّ routine paediatric out-patient clinic were assessed by paediatric nurses; 28 of these children (aged 3 months to 8 years) were also assessed by a single middle grade junioro paediatrician.

Group 2: 78 children (aged 9 days to 10 years) presenting consecutively to the Accident and Emergency (A\&E) department were similarly assessed by experienced $A \& E^{-}$ nursing staff. None of the nurses had specific paediatric training.

Group 3: 24 children (aged 1 month to 9 years) seen consecutively but subsequently too groups 1 and 2, in the A\&E department, were similarly assessed by junior A\&E Senior House Officers.

Analysis for each of the 3 groups was carried out using tape estimated weight, observer estimated weight, actual weight and surface area. Pearson product-moment correlation co-efficients were calculated for each pair of variables using linear regression? analysis. Paired T-tests were performed on the nurse and tape estimates in groups 1 and 2 combined, in order to compare these with the actual weight.

The error $E$ as represented by the formula: $E=\frac{T-0}{T}$, where $T$ is true weight and 0 is estimated weight, was compared for observer and tape predicted weights by paired $\mathrm{T}-\stackrel{0}{\rightarrow}$ tests. The significance of the variance ratio $F$ was predicted from standard tables. 


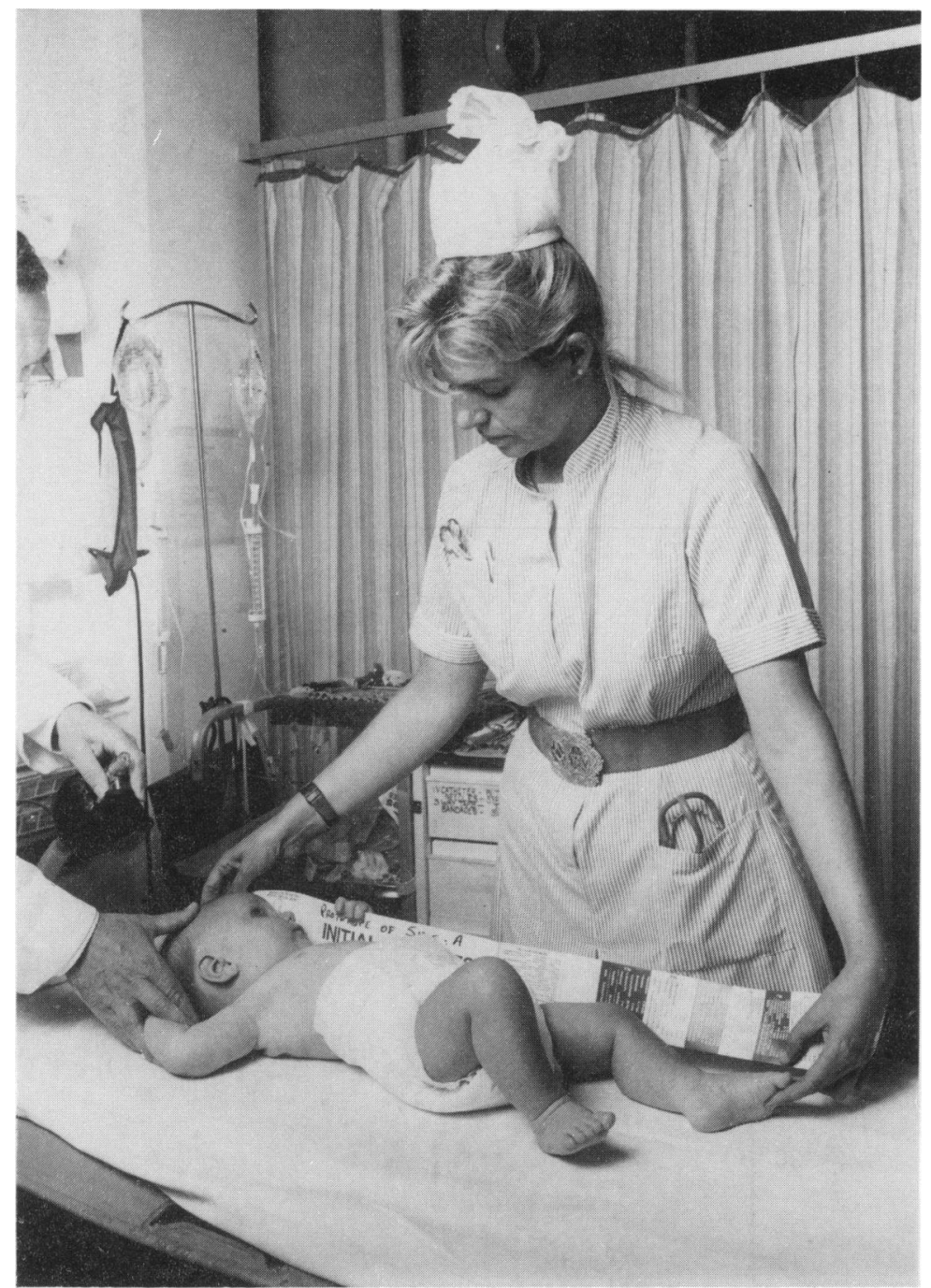

Fig. 1 The tape as used.

\section{RESULTS}

There is a clear correlation of tape estimated weights with true weights $(r=0.974)$ and body surface area $(r=0.983)$ in 139 children aged from 1 week to 10 years.

The correlation co-efficients using paediatric and casualty nurse-estimated weights versus true weights were similar $(r=0.905$ and 0.915 respectively). When the two groups were combined $(n=139)$ a strong correlation co-efficient $(r=0.974)$ between tape estimated and true weights was found (Figure 2). This compared to $r$ values of 
24 G. Hughes et al.

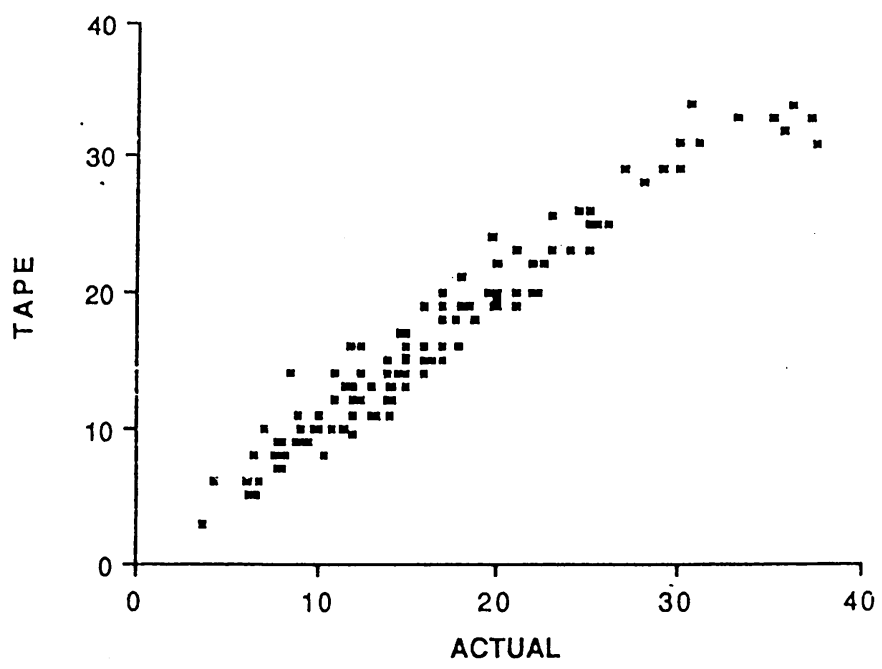


0.907 for nurse estimates (138 cases), 0.915 for paediatrician estimates ( 28 cases) and 0.892 for casualty doctors estimates ( 24 cases) versus the true weight.

\section{Paired T-tests}

138 nurse estimates $-v$ - true weights:

Difference of the mean:

Standard deviation of the difference from the mean:

139 tape estimates - $v$ - true weights:

Difference of the mean:

Standard deviation of the difference from the mean:

In both nurse and tape estimates there is no systematic error tendency to over or underestimate although the greater errors occur in the older and heavier children in both groups (Figure 3); but, because the accuracy of the estimates is based on S.D. of the differences from the mean, it can be seen that the random error is greater with the nurse estimates.

According to the formula:

$95 \%$ range of discrepancies $=1.96 \times$ S.D.

For the nurse-estimated weights:

$\begin{aligned} 95 \% \text { range } & =1.96 \times 3.05 \\ & =6.480\end{aligned}$

i.e., $95 \%$ of true weights were within +6.383 and $-6.574 \mathrm{~kg}$ of the nurse estimated weights.

For the tape-estimated weights:

$$
\begin{aligned}
95 \% \text { range } & =1.96 \times 1.706 \\
& =3.340
\end{aligned}
$$

i.e., $95 \%$ of true weights were within +3.323 and $-2.257 \mathrm{~kg}$ of the tape estimated weights.

From the above calculations, it can be deduced that the use of the tape reduces the random error in the nurse estimates by $51 \%$.

To compare the discrepancies in the nurse estimates and the tape estimates a variance ratio (= (larger S.D. $)^{2} /(\text { smaller S.D. })^{2}$ ) was calculated, obtaining a value of $F=3.753$. This has a null hypothesis $P<0.001$, showing that the variance using the tape was significantly smaller than that found with the nurse estimates.

\section{DISCUSSION}

The sensitivity of infants and young children to errors in drug dosages, particularly to those drugs with low therapeutic index, is greater than that in adults. This is due to the rapidly changing body weight, drug elimination capabilities and different body composition in children of increasing age (Hager et al., 1977; Turner \& Richens, 1978; 

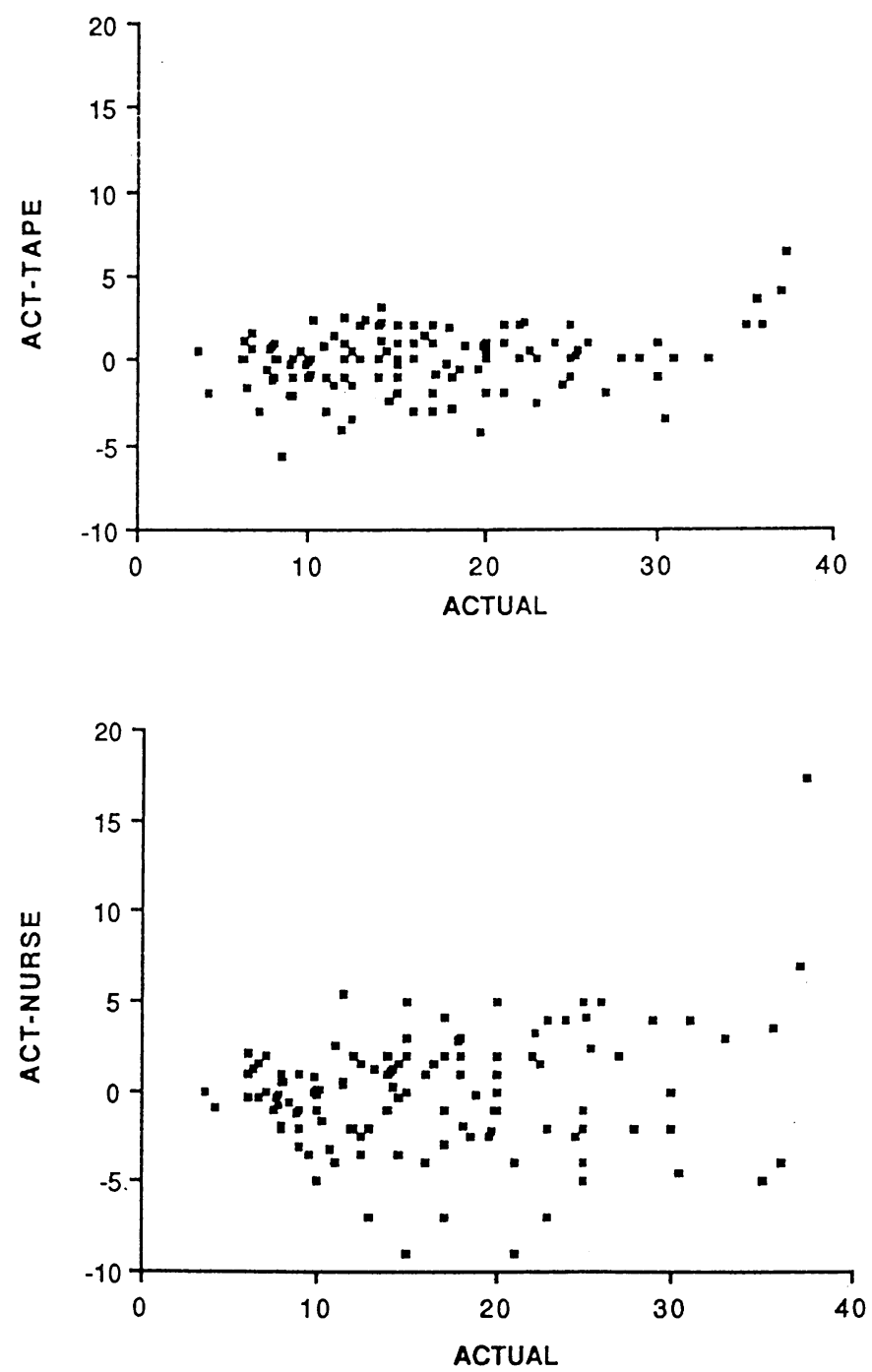

FIGURE 3 : (Numbers represent weight in kilograms)

Fig. 3 Comparison between actual minus tape weight (i.e., tape error) and actual weight (tape graph), and actual minus nurse estimated weight (nurse error) and actual or measured weight (bottom graph).

Abernethy et al., 1982). Of the various methods which have been advocated for drug dose calculation in children the $\mathrm{mg} / \mathrm{kg}$ technique is simple and mostly adequate in the emergency situation.

Because of the difficulties in weighing children when urgent resuscitation is required, there is a real need for a simple method of estimating weight. 
The tape described in this study, by being weight for length graded permits a child's weight to be estimated from measuring its length.

This present study shows it to be 'reliable' in inexperienced hands, significantly reducing the margin of error by $51 \%$ in nurse estimates $(p<0.001)$ and probably more so with $A \& E$ senior house officer estimates, considering the poor correlation in their estimates with true weights.

We did not look at body habitus or sex independently, although the tape-predicted weights will presumably be less accurate in obese or malnourished children. However, our study included a spectrum of children ranging from the well-nourished out-patient visitor to patients under hospital investigation for failure to thrive or oncological disease. We would suggest that, in the case of extreme obesity or emaciation the tapegenerated estimates could be adjusted accordingly, by the observer, to take account of the child's body habitus.

Since our study was completed Oakley (in 1988) using a questionnaire method proposed a paediatric resuscitation chart, which interestingly includes a length system to estimate drug dosage. We believe the tape for length measurement to be a useful adjunct to the emergency management of children, particularly by inexperienced personnel.

\section{ACKNOWLEDGEMENTS}

We would like to thank Dr J. Broselow for providing the tape measure, Tariq Rasheed for computer assistance and Dr K. D. MacRae for statistical advice.

\section{REFERENCES}

Abernethy D. \& Greenblatt D. (1982) Pharmacokinetics of drugs in obesity. Clinical Pharmacokinetics, 7, 108-24.

Catzel P. \& Oliver R. (1981) The Paediatric Prescriber. 5th Edition, pp. 986-9. Blackwell Scientific Publications, Oxford.

Garland J. S. (1986) A rapid and accurate method of estimating body weight. American fournal of Emergency Medicine 5, 390-3.

Hager A., Sjostrom L., Avidson B. (1977) Body fat and adipose tissue cellularity in infants: a longitudinal study. Metabolism 26, 607-14.

Hamill D., Drizd T., Johnson C., Reed, M., Roche, A. \& Moore, W. (1970) Physical growth: National centre for health statistics percentiles. American fournal of Clinical Nutrition 32, 607-29.

Hume R. \& Weyers E. (1971) Relationship between total body water and surface area in normal and obese subjects. Fournal of Clinical Pathology 24, 234-8.

Oakley P.A. (1988) Inaccuracy and delay in decision making in paediatric resuscitation, and a proposed reference chart to reduce error. British Medical fournal 297, 817-19.

Skirkey H. A. (1975) Pediatric Therapy. The C.V. Mosby Company, St. Louis, U.S.A. pp. 19-33.

Standards and guideline for cardiopulmonary resuscitation and emergency cardiac care. JAMA 1986 255, 21, 2961-1969.

Turner P. \& Richens A. (1978) Clinical Pharmacology, pp. 8-24. Churchill-Livingstone, Edinburgh.

Weech A. A. (1956) Signposts on the highway of growth. American fournal Disease of Children 88, 542-7. 